\title{
用高能量密度等离子体在室温下 制备氮化钛薄膜 *
}

间鹏勋 杨思泽 李 兵 陈熙琛

(中国科学院物理研究所, 北京 100080)

\section{关键词高能量密度等离子体、舅化钛薄膜、GCr15 钢}

氮化钛薄膜具有很多优异的性质, 如高硬度, 耐磨性, 耐腐蚀性以及漂亮的金黄色 ${ }^{[1-3]}$. 它 是唯一在工业上被广泛运用的镀膜材料. 然而目前主要沉积氮化钛膜的技术, 如化学气相 沉积 (CVD), 物理气相沉积 (PVD) 和近几年发展起来的离子束技术 (IBM, IBAD) 都有其自 身难以克服的缺点, 它们限制了氮化钛薄膜沉积技术的应用范围. 这些缺点是: 反应温度太 高, 镀膜与基底结合不牢, 以及沉积速率太低 ${ }^{[4]}$.

本文介绍一种全新的氮化钛膜的合成技术 - 高能量密度等离子体技术. 运用该方法, 在 室温下氮化钛薄膜被成功地形成于 GCr 15 轴承钢的表面. 镀氮化钛膜的样品表面显微硬度 比基底材料要高两倍多,并且锫膜与基底材料的粘接性大大增强.

\section{1 实 验 方 法}

产生高能量密度等离子体的装置是我们首次在国内研制的, 装置如图 1 所示. 它由四部 分组成：快速脉冲电磁阀、同轴等离子体枪、充放电电路和真空靶室. 装置的描述也可见文 献[5]. 高能量密度等离子体束产生的过程如下: 首先由真空洜将系统抽到高真空 $\left(10^{-3} \mathrm{~Pa}\right)$, 并将二组储能电容器 $C_{\mathrm{p}}$ 和 $C_{\mathrm{g}}$ 分别加上所需的电压, 再打开脉冲电磁蔺将所需的工作气体快 速充人同轴等离子枪内, 然后通过触发式或 自击穿使其电离, 同时同轴枪的内电极也将 被浌射出一定数量的离子. 电容器 $C_{\mathrm{g}}$ 放电 在内外电极间产生径向电流 $J_{\mathrm{r}}$ 和感应角向 磁场 $B_{\mathrm{e}}$, 在相应的罗仑兹力 $J_{\mathrm{r}} \times B_{\mathrm{e}}$ 作用下, 脉冲等离子体沿轴向被加速并喷出枪口, 最后轰击到样品表面上, 形成所需要的各种 薄膜. 薄膜的种类决定于工作气体和电极 材料. 该装置产生的等离子体密度 $n=$ $10^{14}-10^{16} \mathrm{~cm}^{-3}$; 等离子体温度 $T=10-100$

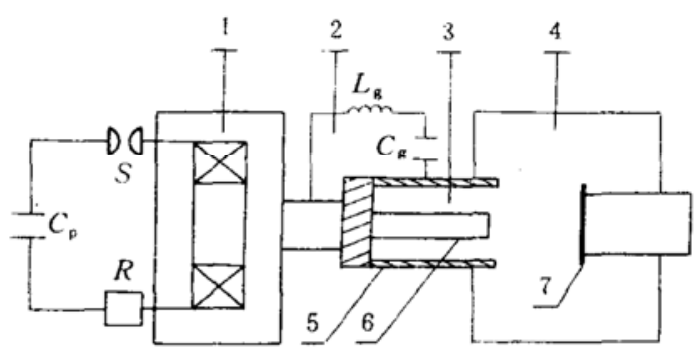

图 1 高能量密度等离子体发生装置示意图 1 为快速脉冲电磁阔, 2 为充放电回路, 3 为同轴等离子体 枪, 4 为真空靶室, 5 为等离子体枪外电极, 6 为等离子体 枪内电极. 7 为样品

1994-01-11 收稿, 1994-04-01 收修改稿.

* 国家自然科学基金和中关村联合测试基金资助项目. 
$\mathrm{eV}$; 等离子体脉冲宽度 $\tau \approx 60 \mu \mathrm{s}$; 等离子体定向速度 $V=(10-50) \mathrm{km} / \mathrm{s}$.

本试验中, 工作气体用纯度 $99.9 \%$ 的氮气, 同轴等离子体枪的内电极为纯钛, 外电极为石 墨碳. 内外电极的电压 $V_{\mathrm{g}}=3 \mathrm{kV}$, 氮气气库的压力 $P_{\mathrm{N} 2}=0.12 \mathrm{MPa}$, 靶室真空度为 $P_{\mathrm{a}}=10^{-3} \mathrm{~Pa}$, 样品材料为抛光的 GCr15 轴承钢. 用扫描电镜, 扫描俄歇探针, $\mathrm{X}$ 光衍射仪, 显微硬度计对形 成的氮化铁膜给予了分析.

\section{2 结 果 与 分 析}

图 2 显示的是在 GCr15 钢表面上形成的氮化钢膜的照片. 图 2(a) 是脉冲等离子体轰击 一次下的情况. 该样品表面呈金黄色. 照片上显示出现大小为 $1-2 \mu \mathrm{m}$ 的晶粒. 并且沉积 膜表面均匀, 未见有大的孔洞与其它缺陷. 图 2(b) 显示的是脉冲等离子体轰击样品 20 次情况 下的形貌, 其显微结构与图 2(a)有所区别, 表面有许多平行的条状区域, 这是位错所引起的.

为了解沉积膜的结构及物相情况, 用掠角人射 $X$ 光衍射仪测量了样品表面. 图 3 显示, 表面膜主要有面心立方的氮化钛结构, 二个衍射峰分别对应于 (111)晶面和 (200)晶面. 这说明

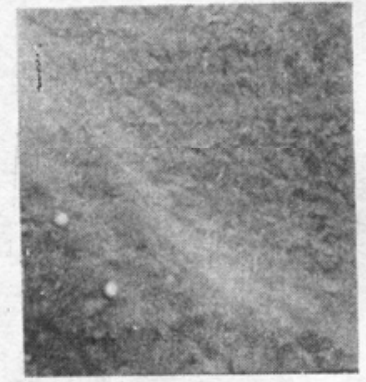

$93122325 \mathrm{kV} \times 1000 \quad 30 \mu \mathrm{m}$

(a)

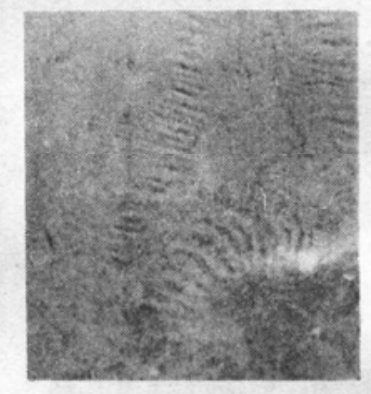

$93122625 \mathrm{kV} \times 1000 \quad 30 \mu \mathrm{m}$

(b)

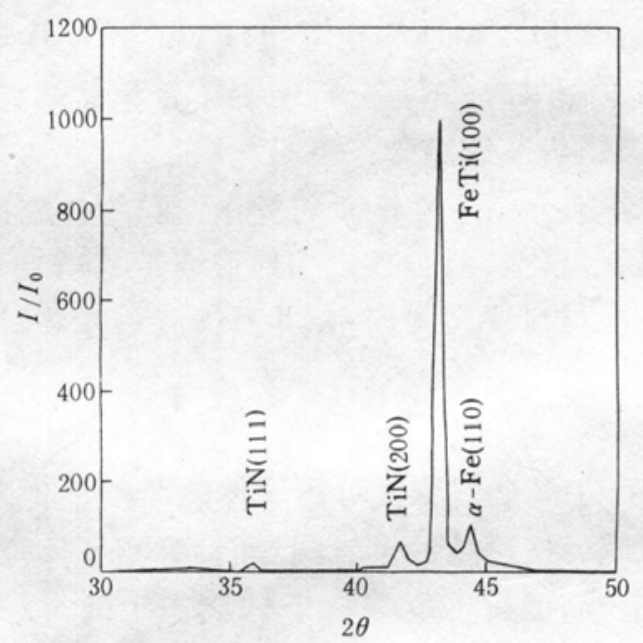

图 3 沉积氮化针膜的 $\mathrm{GCr} 15$ 钢样品的 X光 衍射花样 1 次等离子体束轰击
图 2 GCr 15 钢表面沉积氮化钛膜的电镜形貌

(a) 1 次等离子体束轰击, (b) 20 次等离子体束毒击

经脉冲等离子体束轰击后, GCr15 轴承钢表面形成了多晶的氮化钛薄膜. 衍射图上的最强峰 对应于铁钋合金, 它们存在于沉积膜与 GCr15 钢的混合界面处. 这可由深度成分分析证实.

用 Perkin-Elemer PHI610 扫描俄歇探针 (SAP) 测量了薄膜的化学成分在深度方向上的分 布. 如图 4 所示, 沉积膜与基底有一个宽的混合界面, 界面处主要是铁钛合金, 这使得沉积膜 与基底之间有一个平缓的成分及结构过渡. 它将增强沉积的氮化钢膜与基底的粘结力. 宽的 混合界面归因于高能量等离子体束的注人效应. 这正是本技术的主要特点之一. 表面膜的成 分除了氮原子和钝原子外, 还有一定量的碳原子存在. 这来源于等离子体枪外电极的浌射. 但在该样品的 $\mathrm{X}$ 光衍射图上未发现有碳的化合物存在. 高能量脉冲等离子体轰击 1 次和 2 次样品的沉积膜厚有二倍的关系, 这与台阶仪测出的膜厚也相符. 1 次轰击样品的膜厚约为 $1.5 \mu \mathrm{m}, 2$ 次轰击的样品表面膜厚为 $2.7 \mu \mathrm{m}$.

一些沉积了氮化钢膜的 GCr15 样品的表面显微硬度值列于表 1 中. 沉积有氮化钝膜 


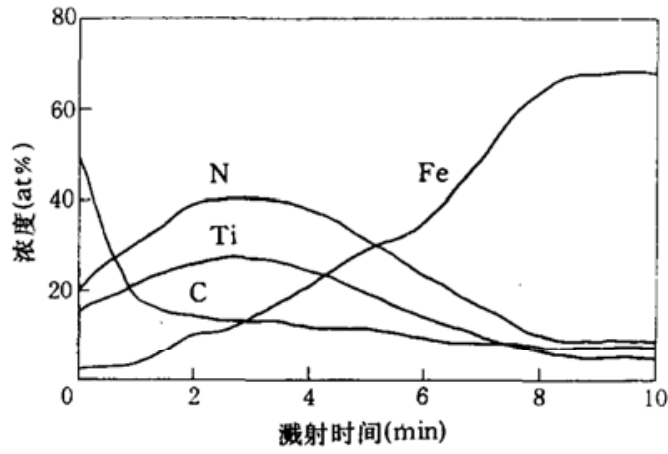

(a)

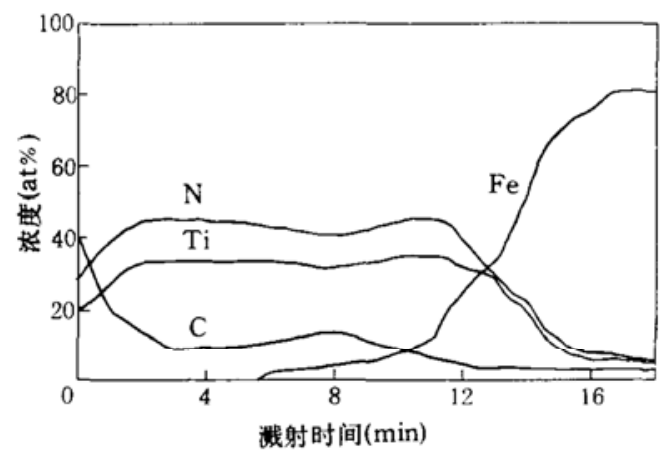

(b)

图 4 等离子体沉积氮化琙膜样品的俄歇深度分布图

(a) 1 次等离子体束轰击, (b) 2 次等离子体束轰击

表 1 高能量密度等离子体在 $\mathrm{GCr} 15$ 钢表面形成的瓷 化针膜的显微硬度 $\left(H_{\mathrm{v}}\right)$ (载荷为 $40 \mathrm{~g}$, 保载时间 $30 \mathrm{~s}$ )

\begin{tabular}{|c|c|c|}
\hline 样品 & 等离子体束轰击次数 & 维氏硬度 $\left(H_{\mathrm{v}}\right)$ \\
\hline $\mathrm{GCr} 15$ 钢 & 0 & 907 \\
\hline No. 1 & 1 & 2216 \\
\hline No. 2 & 2 & 1952 \\
\hline No. 3 & 8 & 2108 \\
\hline No. 4 & 20 & 1908 \\
\hline
\end{tabular}

的 $\mathrm{GCr} 15$ 钢表面的显微硬度比 $\mathrm{GCr} 15$ 钢 有相当大的提高. 平均提高幅度都有二 倍多. 硬度提高的主要原因就是具有高 硬度氮化钝膜的形成. 虽然不同次数的等 离子体束轰击使沉积膜的厚度不同. 但它 们彼此的显微硬度则差不多.

高能量密度等离子体束具有高的电 子温度、电子密度以及高的定向速度. 这

使得离子具有较高的势能和动能. 高的势能有利于室温下一些化合物的形核和长大. 高的 动能能使离子具有注人效应, 这对增强镀膜与基底的粘结性非常有利. 因此用该技术形成的 氮化钝膜克服了传统技术沉积氮化钛膜的缺点, 将扩展氮化针膜的使用范围. 由于高能量密度 等离子体的这些特点, 使它在基底材料表面能产生微观的高温、高压状态, 而整个材料本身还 处于室温状态下. 这将为室温下合成金刚石薄膜和氮化矹薄膜提供了可能性.

\section{3 结 论}

1. 在室温下, 用高能量密度等离子体束, 在 $\mathrm{GCr} 15$ 钢表面成功地制备了性能优良的氮化 钧薄膜：

2. 沉积氮化钝膜的 GCr15 钢表面显微硬度比基底提高两倍多, 并且沉积膜与基底有较宽 的混合界面, 这将大大增强膜与基底的粘结性.

3. 沉积的薄膜主要由多晶的面心立方结构氮化钛所组成. 界面处有铁钛合金的生成.

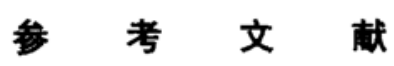

[1] Bonelli, M., Guzman, L. A., Miotello, A. et al., Vacuum, 1992, 43(5-7): 459.

[2] Chatterjee, S., Chandrashekhar, S., Sudarshan, T. S., J. Materials Science, 1992, 27: 3409.

[3] Sundgreen, J. E., J. Vacuum Science Technology (A), 1985, 3(3): 614.

[4] Yan, P. X., Physica Status Solidi, 1991, A124: 483.

[ 5] Shen, Z. G., Lee, C. H., Wu, C. et al., J. Materials Science, 1990, 25: 3139. 